UNDERGRADUATE RESEARCH IN NATURAL AND CLINICAL SCIENCE AND TECHNOLOGY (URNCST) JOURNAL Read more URNCST Journal articles and submit your own today at: https://www.urncst.com

\title{
Evaluating the Effectiveness and Efficiency of a Laser Guided and Electrodynamic Approach to Performing Space Debris Removal
}

\author{
Manish Dev Angrish, BSc Student [1]*, Matthew Badal-Badalian, BEng Student [2], \\ Edward Shen, BSc Student [3]
}

[1] Department of Biochemistry and Biomedical Sciences - Biomedical Research Specialization, McMaster University, Hamilton, Ontario, Canada, L8S 4L8

[2] Department of Electrical and Computer Engineering, McMaster University, Hamilton, Ontario, Canada, L8S 4L8

[3] Department of Biochemistry and Biomedical Sciences, McMaster University, Hamilton, Ontario, Canada, L8S 4L8

*Corresponding Author: angrishm@mcmaster.ca

\begin{abstract}
Introduction: Space debris is an alarming problem that researchers around the globe have been working to solve. These space particles consist of meteorites that exist in space as well as fragmentation originating from satellites and spacecraft. The effect of these debris can be devastating upon collision with devices such as satellites, telecommunicators, etc. The National Aeronautics and Space Administration database reported that approximately 200 million objects were found orbiting close to the Earth. Currently, different techniques are under investigation to eliminate space debris.

Methods: Space debris varies in size, thus can only be fully removed if the various sizes are accounted for. We propose a model where efficiency and effectiveness can be optimized towards certain sizes of space debris. The laser ablation process involves concentrating multiple laser pulses on space debris to create plasma plumes which eject from the cavity created in the material at a substantial velocity, acting against the material's original orbit and can be guided into Earth's atmosphere where it will disintegrate or fall into the ocean. An electrodynamic net will also be used to attract and capture space debris. Once taken a hold of, this debris will be transported by the satellite towards the Earth's atmosphere. Here both the satellite and the debris will be disintegrated.

Results: To solve this problem, this study proposes a dual-debris model which combines a laser-guided and electrodynamic approach. The first process involves short-wavelength laser pulses that focus on debris greater than $10 \mathrm{~cm}$ in diameter, generating plasma plume ejections that decrease orbital velocity. The electrodynamic approach will attract and dispose materials less than $10 \mathrm{~cm}$ in diameter with an electrically charged net dispatched by a satellite, which is then guided towards the Earth.

Discussion: Based on the results of the studies analyzed, laser ablation is an effective method of removing large space debris while the electrodynamic net serves as a cost-efficient method of eliminating small debris. Both methods allow targeted debris to approach and disintegrate in the Earth's atmosphere or fall into the ocean. Results from previous studies were largely based on simulations, and further testing should be done.
\end{abstract}

Keywords: space debris; electrodynamic; laser; space exploration; satellites; atmosphere

\section{Introduction}

Space exploration has been a topic of interest for many decades, however, there are many obstacles that need to be overcome in order to ensure the safe transportation of our astronauts and other technological devices. One of the most pressing matters of late has been the recognition of millions of space debris particles surrounding the Earth's atmosphere. These space particles can consist of meteorites that naturally exist in space. However, this does not compare to the number of artificial parts, such as the fragmentation originating from satellites and spacecraft [1].
When space vehicles are launched, many small objects stay in the Earth's low orbit for a couple of years before burning up in the atmosphere. On the contrary, if debris is left at altitudes higher than $36,000 \mathrm{~km}$, it can become trapped in the Earth's orbit for thousands of years [1]. These space particles provide hindrances to many facets of space exploration and technological machines, as they can travel up to speeds of $28,175 \mathrm{~km} / \mathrm{h}$ [2]. The dead satellites and rocket parts orbiting the Earth could soon cause a rippling effect known as Kessler Syndrome, where one crash could produce an infinite amount of collisions. This would, in 
UNDERGRADUATE RESEARCH IN NATURAL AND CLINICAL SCIENCE AND TECHNOLOGY (URNCST) JOURNAL Read more URNCST Journal articles and submit your own today at: https://www.urncst.com

turn, make the Earth's orbit unusable and put an end to all current and future space programs. According to NASA, there are currently 27,000 pieces of space debris that can be detected by the Department of Defense's global Space Surveillance Network sensors, however, they estimate there to be many more objects orbiting the Earth, as smaller sized debris can be hard to track [2]. Since space debris can dramatically vary in size, a dual approach method has been proposed. A laser-mediated method would target objects greater than $10 \mathrm{~cm}$ in diameter, whereas an electrodynamic net approach would target objects less than $10 \mathrm{~cm}$ in diameter. This study seeks to evaluate the effectiveness and efficiency of laser ablation and electrodynamic nets as methods of eliminating space debris of varying sizes.

\section{Methods}

Laser ablation: Shen et al. [3], explores the optimal choice of laser transmission by taking into consideration four conditions that should be met: high average power and peak power, high pulse energy, high laser beam quality, and mature technology with simple maintenance [3]. The authors describe a reliable solid-state laser operating in a burst mode that does not have a mechanically driven component and no need to refuel the system [3]. They determine the optimal mirror diameter to be $2.44 \mathrm{~m}$ to obtain a $15 \mathrm{~cm}$ focal spot considering the difficulty of tracking and energy waste [3]. They also demonstrate that the optimal laser repeat frequency to be $100 \mathrm{~Hz}$ assuming laser pulse energy is $1 \mathrm{~kJ}$ and considering effectiveness and cost [3]. The mass is the most important parameter when studying the motion of space debris [3]. Through the statistical analysis, it was found that the mass of space debris is proportional to the diameter of the debris, raised to the power of $2.26\left(\mathrm{~m} \propto \mathrm{d}^{2.26}\right)$ [3]. Space debris is mainly composed of aluminum and carbon, which follow the same model [3]. Thus, the properties of these elements are very useful for understanding space debris [3]. Using the same model, debris with a diameter of $10 \mathrm{~cm}$ would weigh $70 \mathrm{~g}$ if it was purely made out of aluminum and $40 \mathrm{~g}$ if it was purely carbon-based [3]. A mechanical impulse, $\mathrm{m} \Delta \mathrm{v}$, of a material, is equivalent to the coupling coefficient, $C_{m}$, multiplied by the incident laser pulse energy, $\mathrm{E},(\mathrm{m} \Delta \mathrm{v}=$ $\mathrm{C}_{\mathrm{m}} \mathrm{E}$ ) [3]. The coupling coefficient, $\mathrm{C}_{\mathrm{m}}$, is found to be $1.4 \times$ $10-5 \mathrm{~N} \cdot \mathrm{s} / \mathrm{J}$ for carbon and $2 \times 10-5 \mathrm{~N} \cdot \mathrm{s} / \mathrm{J}$ for aluminum according to experimental results [3]. However, the coupling coefficient, $\mathrm{C}_{\mathrm{m}}$, only remains constant when the wavelength, intensity, and pulse length of the laserirradiated to the debris material also remain constant [3].

Soulard et al [4]. describe the laser requirements for the removal of space debris [4]. For aluminum debris with an initial orbit of $800 \mathrm{~km}$, the orbital velocity would need to be decreased by $2.5 \%$ in order for it to reach an altitude of 100 $\mathrm{km}$ where re-entry into the atmosphere occurs [4]. Additionally, it was found that laser emission in spacebased operations was more energy efficient compared to ground-based operations [4]. This study distinguishes regimes in size, energy, and pulse duration between space and ground-based operations, noting that nanosecond pulse durations would be suitable for atmospheric transmission, and shorter picosecond pulses would be suitable for space transmission [4]. This study also outlines a novel architecture, the International Coherent Amplification Network (ICAN) project, a space-based operation that is capable of tracking and de-orbiting hyper-velocity space debris [4].

Electrodynamic net: In a study conducted by Pearson et al. [5], the authors discuss their spacecraft, ElectroDynamic Debris Eliminator (EDDE), and the various benefits it provides. In a simulation conducted to remove 2,465 objects in Low Earth Orbiting weighing greater than $2 \mathrm{~kg}$, EDDE was compared with other propulsion systems [5]. The outcomes showed that an estimated 12 EDDEs were required to fulfill this task, compared to the next closest of 30 vehicles using the Variable Specific Impulse Magnetoplasma Rocket (VASIMR) propulsion system. Moreover, the estimated total mass in orbit was $0.04 \%$ of the VASIMR system. Furthermore, it was hypothesized 12 EDDEs could get rid of 2,166 tons worth of space debris under a 7-year time span. Alongside this, Pearson et al. [5] also discuss the versatility of their net dispenser, as it would be a $100 \mathrm{~m}$ tether that would have the ability to both project forward and be reeled backwards, allowing for the abortion of any poorly executed launch and correction to any mistakes that were made [5].

In another study performed by Sharf et al. [6], the closing mechanism of the tethered net was explored. The researchers decided to use a lump-parameter model, where the mass of the net consists primarily of a finite number of physical knots of the net, and the axial stiffness properties are represented by massless springs between the point masses [6]. When the net is launched, it has minimal tension, however when the net starts to initiate closure, it reaches maximum tension $(\sim 10 \mathrm{~N})$. The speed of the tether in the stimulation was less than $2 \mathrm{~m} / \mathrm{s} \mathrm{[6].} \mathrm{The} \mathrm{experiment}$ conducted by this researchers lasted 100 seconds, and had 6 steps within that timespan: (a) net starts deploying $(\mathrm{t}=1 \mathrm{~s})$, (b) net and debris are in contact $(\mathrm{t}=17 \mathrm{~s})$, (c) closing mechanism is activated and tethers are shortened $(\mathrm{t}=33 \mathrm{~s})$, (d) debris is captured $\mathrm{t}=40 \mathrm{~s}$, (e)-(f) capture is sustained ( $\mathrm{t}$ $=78$ seconds and $\mathrm{t}=100$ seconds, respectively) [6]. The closing mechanism of the net revolved around the idea that the tether would shorten around the net's perimeter, which would thereby allow the net to close around the target [6].

In addition, in a study performed by Bevenuto et al [7]. several ideas for different aspects of the tether net deployment are mentioned. In terms of collision detection, the researchers propose to use a hierarchical boundingboxes collision detection algorithm called Minimum Spherical Bounding Boxes. The net that Bevenuto et al [7]. felt could be effective is one of a planar or pyramid shape and can be either interlaced or non-interlaced. The planar net and 3-D pyramid-shaped nets would have differences in 
mass, mesh length, closing time and target size, all of which would be greater for the former [7]. The authors also discuss post-burn as a crucial aspect of the process. According to the researchers, the most effective way to carry out this procedure would be to produce a single burn and then cut the tether and perform a collision-avoidance maneuver [7]. As well as this, the stiffness of the tether was also considered in-depth, with the conclusion pointing to a more stiff tether being more advantageous. This is apparent as a non-stiff tether makes the chaser attitude more difficult to control and the drift from velocity direction alignment is greater, meaningless transfer efficiency, as well as generating the possibility of creating entanglements [7].

\section{Results}

Laser ablation: This protocol includes a dual debris model. The first approach involves guided laser pulses targeted for space debris greater than $10 \mathrm{~cm}$ in diameter. Technologies relevant to this approach are easily accessible such as steering optics, monitoring and tracking, optics, and laser technologies [8]. The laser ablation process involves multiple laser pulses to create plasma plumes resulting from laser detonation [9]. The laser will use a short ultraviolet wavelength since it is most effective in laser-materials interactions and the pulse duration can vary between milliseconds to femtoseconds [9]. Laser ablation is preferable since short laser pulses remove space material so frequently that surrounding material heats up from absorbing laser energy [9]. The compressed pulse power is high enough to melt and vaporize the material and generates plumes of plasma which eject from the cavity created in the space material [9]. The plasma plumes eject at a substantial velocity of $10^{5} \mathrm{~m} / \mathrm{s}$ in a vacuum, acting against the material's original orbit velocity, as seen in Figure 1 [9]. With enough repeated laser detonations, the debris can be guided into Earth's atmosphere where it will disintegrate or fall into the ocean.

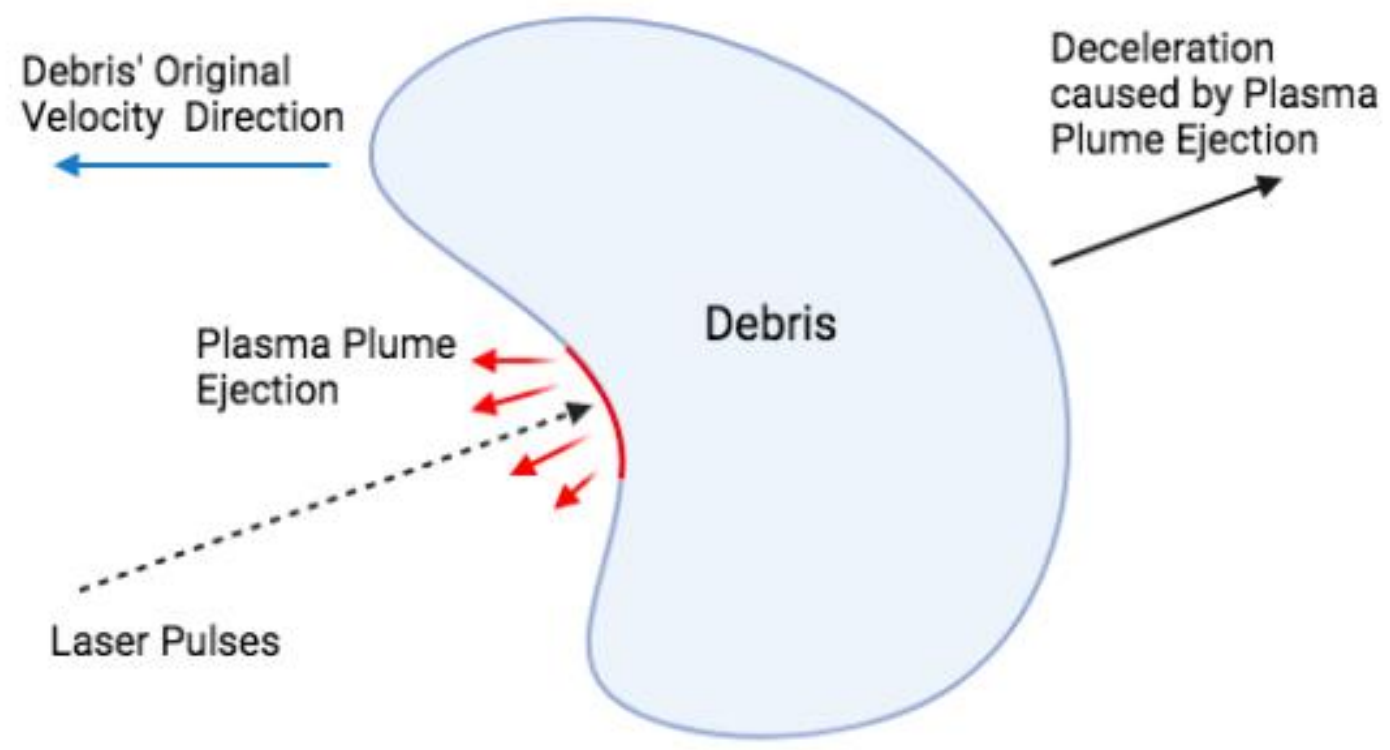

Figure 1. Vector model of plasma plume generation upon laser ablation of space debris material. Plasma plumes are ejected from the cavity created by a train of laser pulses on space debris. The ejection of plasma plumes creates a force that acts against the debris's original motion, and causes the debris to change direction and decelerate [3]. Created via Biorender.

Electrodynamic net: The second approach includes an electrodynamic net used to attract and capture space debris under $10 \mathrm{~cm}$ in diameter. This net will be electrically charged, allowing it to create electrostatic and inductive interactions with space debris. Since most space debris has a metallic component to it, this attraction would be enhanced, allowing for an effective method of collecting the debris. The net itself would be light in weight ( 50 g) for easy transportation - therefore, not creating an overwhelming load for their respective corresponding satellites. The satellite will have the ability to navigate its own motion and position by adjusting to different sets of angles in order to perform the launch of the electrostatic net. The net will be launched in a "harpoon-like manner" with a velocity of $5-10 \mathrm{~km} / \mathrm{s}$ [10]. The net will still be attached to the satellite, however, it will have the ability to extend out to about $10 \mathrm{~km}$ in length, as seen in Figure 2. These nets would have the prototypical dimensions of $40 \mathrm{x}$ 50 × $60 \mathrm{~cm}$, but could also be made with the slight alteration of 5-10 cm to allow for adaptability depending on the circumstance. These dimensions will allow for an effective method of collecting debris less than $10 \mathrm{~cm}$ in 
diameter and will have an overall volume of $\sim 0.120 \mathrm{~m}[8]$. As described by Sharf et al. [6], the net is composed of four separate tethers which are all reeled on the same winch, and each passing through a ring at the central node of the net, then through a ring at a corner node on the net and a ring at the central node on a side of the net. Finally, each tether is attached to the next corner node, with respect to the corresponding "ringed" corner of the net [6]. Once the debris is taken hold of (through electrostatic interactions), this net will be transported via the satellite towards the
Earth's atmosphere. Here, both the satellite and the debris will be disintegrated. In order to make this whole process much more efficient, the satellites will include net managers - which will contain $\sim 100$ nets - from which the nets will be extended from due to centrifugal force at the end [10]. This will allow for more effective use of each satellite - not only increasing the capacity of each satellite, but also limiting the amount of waste product being produced.

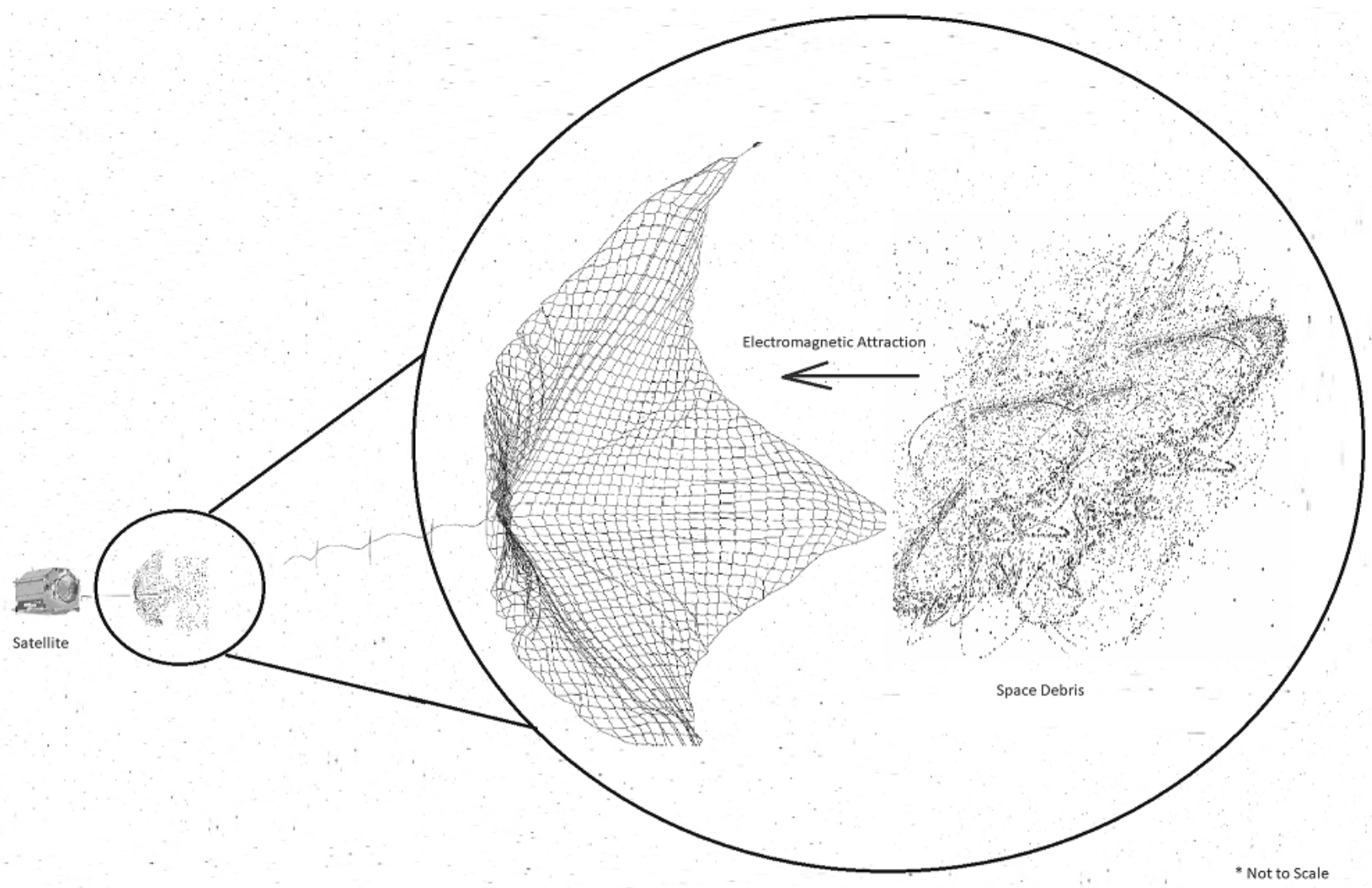

Figure 2. Magnified view of the electrodynamic net being launched in a harpoon-like manner. The satellite launches an electrodynamic net towards the targeted space debris. Using electrostatic interactions, the space debris will be captured by the net, upon which the net will close. From there, the satellite will de-orbit, directing both the net and itself towards disintegration via the Earth's atmosphere. Created via Microsoft Paint, and inspired by the Cable News Network [10]

\section{Discussion}

Laser ablation: The laser transmission parameters discussed in the study by Shen et al. [3], demonstrate detailed and sophisticated technical knowledge of a potential laser system. The study's proposed solution of a solid-state laser operating in a burst mode which does not have a mechanically-driven component and no need to refuel the system would be reliable and require minimal maintenance. A low-maintenance laser system would be beneficial for space-based operations and have lower costs in the long term. This study also outlines parameters of a laser system such as mirror diameter which would be optimized for balancing the tracking of space debris as well as energy waste, and laser repeat frequencies that would be optimized for laser ablation effectiveness as well as energy costs. The space debris mass model constructed in this study could be useful for tracking the motion of space debris since it is considered as one of the most important parameters. However, this model is limited by debris made up of common materials such as aluminum and carbon.

Space-based operations of laser systems seem to be preferable to ground-based operations as outlined by the 
UNDERGRADUATE RESEARCH IN NATURAL AND CLINICAL SCIENCE AND TECHNOLOGY (URNCST) JOURNAL Read more URNCST Journal articles and submit your own today at: https://www.urncst.com

study by Soulard et al. [4], The authors suggest that energy expenditure would be more efficient in space-based operations, adding distinguishing features including size, energy, and pulse duration between space and ground-based operations. This study features a space-based model for tracking and de-orbiting hyper-velocity space debris, the ICAN project, which could be considered for a space-based model designed for larger space debris (greater than $10 \mathrm{~cm}$ in diameter).

Overall, the mechanism of space debris removal by means of laser ablation and the technologies relevant to this approach are well understood and relatively easily accessible. An important next step to an eventual future of utilizing laser ablation to remove space debris could be the experimentation of the laser parameters and conditions discussed in the previous studies. Further research and results from simulations of the proposed laser systems would verify the proposed solutions made in the studies such as the effectiveness of space-based operation compared to ground-based operation.

Electrodynamic net: The second process that was explored was the use of an electrodynamic net to capture and disintegrate debris less than $10 \mathrm{~cm}$ in radius. Based upon previous studies and their simulations, this approach seems to be quite efficient. These experiments examined the structure and properties of the net, the enclosing mechanism and timing, as well as other key processes involved to carry out this procedure. Using these results, these ideas could also be applied to this study's proposed model. For example, in the study done by Pearson et al. [5], the EDDE propulsion system could be incorporated within the design. This is because based on the simulations done by the research team, this system seemed by far to be most efficient at removing space debris, without having to orbit an enormous amount of satellites [5], therefore, being both economically and ecologically viable.

Alongside this, the net release and capture motion can be similar to that as described by Sharf et al. [6], In the experiment, the researchers went into great detail to discuss the structure of the net, the launch and enclosure process, and tension properties. Since this aspect of the procedure only took 100 seconds and had a maximum tension of $\sim 10$ $\mathrm{N}$ in simulations, this seems to be a very effective and efficient approach to carry out the launch and enclosure of the space debris [6]. Furthermore, using the details from the study performed by Bevenuto et al. [7], the shape of the net that could be pyramidal in shape. This is because the pyramidal-shaped net is much more effective in collecting smaller-sized debris, compared to the planar shape net [7]. Specifically, the planar shape net targeted debris that measured $30 \mathrm{~m}$ x $30 \mathrm{~m}$, whereas the pyramidal shape net targeted space debris that was $1 \mathrm{~m} \mathrm{x} 1 \mathrm{~m}$ in size. In addition to this, as described in the same study, the net would also be made of material that would allow for increased stiffness, to minimize entanglements and other problems. Moreover, the de-orbiting and disintegration processes mentioned in the study should also be explored in greater detail in future studies.

In general, a dual-debris model would maximize strengths and minimize downfalls of laser ablation and the electrodynamic net, as laser ablation could be sparingly used towards large debris due to its effectiveness and high cost while the electrodynamic net could be used against a vast amount of smaller debris.

A key area which future studies could explore in greater detail is the ability of the net to capture debris that is generally smaller in size. Of the studies that were explored, they all had experimental space debris sizes that were far greater than ones that would be $10 \mathrm{~cm}$ in diameter. By performing these types of studies, there would be more information on whether this method would be effective against the debris of all sizes. This in turn would also allow for one to identify whether different methods are needed for variously sized objects, or whether this electrodynamic net would be effective enough to be deployed on its own. Alongside this, another aspect that should be looked into is the type of material that would be used to create the net. There are various types of metals that could be manipulated to give the net its electrodynamic nature, however, each metal would also bring its own physical properties as well. These properties could affect the mass, tension and stiffness of the net, which as indicated by Bevenuto et al. [7], could lead to other situations to arise.

\section{List of Abbreviations Used}

EDDE: electrodynamic debris eliminator ICAN: international coherent amplification network NASA: national aeronautics and space administration VASIMR: variable specific impulse magnetoplasma rocket

\section{Conflicts of Interest}

The authors report no potential conflicts of interest.

\section{Ethics Approval and/or Participant Consent}

This study did not involve human or animal participants. It was a study that proposes a method of space debris removal, by reviewing other related articles and journals.

\section{Authors' Contributions}

MDA: made substantial contributions to the design of the study, the collection of data as well as interpretation and analysis of the data, revised the manuscript critically, and gave final approval of the version to be published.

ES: made substantial contributions to the design of the study, the collection of data as well as interpretation and analysis of the data, revised the manuscript critically, and gave final approval of the version to be published.

MABB: made substantial contributions to the design of the study, the collection of data as well as interpretation and analysis of the data, and gave final approval of the version to be published. 
UNDERGRADUATE RESEARCH IN NATURAL AND CLINICAL SCIENCE AND TECHNOLOGY (URNCST) JOURNAL Read more URNCST Journal articles and submit your own today at: https://www.urncst.com

\section{Acknowledgements}

This study was supported by DeSCIpher: McMaster University Life Sciences Competition.

\section{Funding}

This study was not funded.

\section{References}

[1] What is space junk and why is it a problem? [Internet]The Trustees of The Natural History Museum. 2020. [cited 2021 Jun 10]. Available from: https://www.nhm.ac.uk/discover/what-is-space-junkand-why-is-it-a-problem.html

[2] Space Debris and Human Spacecraft. [Internet] National Aeronautics and Space Administration. 2021. [cited 2021 Jun 11]. Available from: https://www.nasa.gov/mission_pages/station/news/orbi tal_debris.html

[3] Shuangyan Shen, Xing Jin, Chang Hao. Cleaning space debris with a space-based laser system. Chinese Journal of Aeronautics. 2014 Aug 27;(4):805-811. https://doi.org/10.1016/j.cja.2014.05.002

[4] Soulard R, Quinn MN, Tajima T, Mourou G. ICAN: A novel laser architecture for space debris removal. Acta Astronautica. 2014 Dec;105(1):192-200. https://doi.org/10.1016/j.actaastro.2014.09.004
[5] Pearson J, Oldson J, Levin E. ElectroDynamic Debris Eliminator (EDDE): Design, Operation, and Ground Support. Star Technology and Research, Inc. 2010

[6] Sharf I, et al. Experiments and simulation of a net closing mechanism for tether-net capture of space debris. Acta Astronautica. 2017 Oct;139:332-343. https://doi.org/10.1016/j.actaastro.2017.07.026

[7] Benvenuto R, Salvi S, Lavagna M. Dynamics analysis and GNC design of flexible systems for space debris active removal. Acta Astronautica. 2015 Jun;110:247265. https://doi.org/10.1016/j.actaastro.2015.01.014

[8] Assessment Study of Small Space Debris Removal by Laser Satellites. [Internet] NTRS - NASA Technical Reports Server. [cited 2011 Mar 5]. Available from: https://ntrs.nasa.gov/citations/20120009369

[9] Bando T, Abe K, and Yamashita M. Effect of $\mathrm{ZnO}$ Plasma plume dynamics on laser ablation. Optical Review. 2010 Jun 5;17(3):309-312. https://doi.org/ 10.1007/s10043-010-0055-y

[10] Cable News Network. [Internet] Cleaning up space debris. [cited 2021 Jun 28]. Available from: https://www.cnn.com/2017/06/13/tech/gallery/spacesweepers/index.html

\section{Article Information}

Managing Editor: Jeremy Y. Ng

Peer Reviewers: Aishwaria Maxwell, Renee Hu, Jennifer Bae

Article Dates: Received Sep 27 21; Accepted Dec 29 21; Published Jan 1422

\section{Citation}

Please cite this article as follows:

Angrish MD, Badal-Badalian M, Shen E. Evaluating the effectiveness and efficiency of a laser guided and electrodynamic approach to performing space debris removal. URNCST Journal. 2022 Jan 14: 6(1).

https://urncst.com/index.php/urncst/article/view/327

DOI Link: https://doi.org/10.26685/urncst.327

\section{Copyright}

(C) Manish Dev Angrish, Matthew Badal-Badalian, Edward Shen. (2022). Published first in the Undergraduate Research in Natural and Clinical Science and Technology (URNCST) Journal. This is an open access article distributed under the terms of the Creative Commons Attribution License (https://creativecommons.org/licenses/by/4.0/), which permits unrestricted use, distribution, and reproduction in any medium, provided the original work, first published in the Undergraduate Research in Natural and Clinical Science and Technology (URNCST) Journal, is properly cited. The complete bibliographic information, a link to the original publication on http://www.urncst.com, as well as this copyright and license information must be included. 


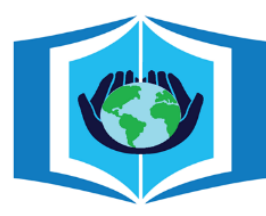

\section{URNCST Journal \\ "Research in Earnest"}

\section{Funded by the \\ Government of Canada}

Canadắ

Do you research in earnest? Submit your next undergraduate research article to the URNCST Journal!

| Open Access | Peer-Reviewed | Rapid Turnaround Time | International | | Broad and Multidisciplinary | Indexed | Innovative | Social Media Promoted |

Pre-submission inquiries? Send us an email at info@ urncst.com | Facebook, Twitter and LinkedIn: @URNCST

Submit YOUR manuscript today at https://www.urncst.com! 